\title{
Determination of Bitterness of Andrographis Herba Based on Electronic Tongue Technology and Discovery of the Key Compounds of Bitter Substances
}

\author{
Xiao Zhang ${ }^{1,2,+}{ }^{\dagger}$ Hongwei $\mathrm{Wu}^{1,+}{ }^{\dagger}$, Xiankuo Yu ${ }^{1}$, Hanyan Luo ${ }^{1}$, Yaqi Lu ${ }^{1,2}$, Hongjun Yang ${ }^{1}$, \\ Xin $\mathrm{Li}^{1}$, Zhiyong $\mathrm{Li}^{3}{ }^{3}$, Liying Tang ${ }^{1, *}$ and Zhuju Wang ${ }^{1, *}$ \\ 1 Institute of Chinese Materia Medica, China Academy of Chinese Medical Science, No.16 Nanxiaojie, \\ Dongzhimennei Ave., Beijing 100700, China; zhangxiaoyx163@163.com (X.Z.); \\ whw9905012@163.com (H.W.); yuxiankuobz@126.com (X.Y.); lhanyan7@163.com (H.L.); \\ luyaqi412@163.com (Y.L.); hjyang@icmm.ac.cn (H.Y.); 18406557239@163.com (X.L.) \\ 2 College of Pharmacy, Henan University of Chinese Medicine, No.156 Jinshuidong Ave., \\ Zhengzhou 450046, China \\ 3 State Key Laboratory of Innovative Natural Medicine and TCM Injections, Jiangxi Qingfeng Pharmaceutical \\ Co. Ltd., No. 8 Zhandongdong Ave., Ganzhou 341000, China; jxlzy2@qfyy.com.cn \\ * Correspondence: bjtangliying@163.com (L.T.); wangzhuju@sina.com (Z.W.); \\ Tel./Fax: (+86)-10-64087609 (L.T.); (+86)-10-64087609 (Z.W.) \\ + These authors contributed equally to this work.
}

Received: 11 December 2018; Accepted: 14 December 2018; Published: 19 December 2018

\begin{abstract}
Andrographis Herba (AH), the dry aerial segments of Andrographis paniculata (Burm.f.) Nees, is a common herbal remedy with bitter properties in traditional Chinese medicine (TCM) theory. Although bitterness is one of the features representing Chinese medicine, it has not been implemented as an index to assess the quality and efficacy of TCM because of peoples' subjectivity to taste. In this study, 30 batches of AH with different commercial classifications (leaves, stems, or mixtures of both) were collected. Bitterness of AH was quantified by electronic tongue technology. Meanwhile, chemical compositions were characterized through establishing high-performance liquid chromatography fingerprints. The result indicated that the radar curves of the bitterness from different $\mathrm{AH}$ commercial classifications displayed different taste fingerprint information. Based on six taste factors, a Principal Component Analysis (PCA) score three-dimensional (3D) plot exhibited a clear grouping trend $\left(R^{2} X, 0.912 ; Q^{2}, 0.763\right)$ among the three different commercial classifications. Six compounds (Peaks 2, $3,4,6,7,8)$ with positive correlation to bitterness were discovered by a Spearman correlation analysis. Peaks 2, 6, 7, 8 were identified as andrographolide, neoandrographolide, 14-deoxyandrographolide, and dehydroandrographolide, respectively. The electronic tongue can be used to distinguish AH samples with different commercial classifications and for quality evaluation.
\end{abstract}

Keywords: Andrographis Herba; electronic tongue; chromatographic fingerprints; Principal Component Analysis; Spearman correlation analysis; bitter substances

\section{Introduction}

Andrographis Herba (AH, Chuan Xinlian in Chinese) is derived from the dry aerial segments of Andrographis paniculata (Burm.f.) Nees, and it has been used in folk medicines for the treatment of fever, common colds, diabetes, hepatitis, skin infections, snake bites, hypertension, and other diseases in several Asian countries, including China, India, and Thailand [1]. Modern pharmacological research has revealed that $\mathrm{AH}$ has multiple properties, including anti-inflammatory, bacteriostasis, antioxidative, antitumor, hypoglycemic, cardiovascular, and hepatoprotective [2]. The major active components of 
$\mathrm{AH}$ are considered to be labdane diterpenoid lactones and flavones [3]. In the Pharmacopoeia of the Republic of China, the medicinal part of $\mathrm{AH}$ was the aerial segments [4]. However, in fact there are three types of AH samples with different specifications available in the Chinese raw herbal medicine market, which are leaves, stems, or a mixture of both.

According to Traditional Chinese Medicine (TCM) theory, the properties and actions of Chinese herbs refer to their nature and effects relating to treatments. These properties include the "five flavors", which are tastes composed of pungent, sweet, sour, bitter, and salty, which are high-level summary of the clinical efficacy [5]. For example, some herbal medicines with a bitter flavor possess properties for drying or resolving dampness, purging, and lowering, and are often used to treat constipation due to fired-heat, dysphoria, cough due to the adverse rising of lung-qi, damp heat, or cold damp syndrome [6]. AH is a representative bitter herb, which has the effect of clearing away inner heat, eliminating toxins, drying dampness, and relieving swelling [4]. In the clinic, $\mathrm{AH}$ is generally believed that, when compared with stems, $\mathrm{AH}$ leaves have better effects. It is subjectively believed that AH leaves are more bitter than stems. Actually, the taste perceived by the mouth is susceptible to physical and psychological conditions, such as differences in the amount of taste buds or personal preferences [7]. Up to now, there were some reports about the quality evaluation of $\mathrm{AH}$ samples with different specification by HPLC and UPLC analysis [8,9]. However, the bitterness of AH samples have not been quantitatively and objectively evaluated, and research is lacking on the relationship between the bitterness and chemical compounds of $\mathrm{AH}$.

The use of electronic tongues offers a promising alternative to solve those problems, which consists of an analytical sensor array system that is able to detect specific substances by means of different artificial membranes and electrochemical techniques [10]. Electronic tongues were initially implemented in food sectors in the 1980s [11,12] and later in various other areas, for example, TCM quality control [13,14], taste masked drug formulations [15-18], and identification of Chinese medicine adulteration [19], as well as for changes in taste before and after TCM processing [20,21].

In this study, electronic tongue technology was firstly used to quantitatively and objectively detect bitterness and evaluate the quality of $\mathrm{AH}$ samples with different commercial classifications. Furthermore, a fingerprint method was developed for characterizing chemical composition, and correlating the chemical composition with bitterness to explore the key of bitter compounds of $\mathrm{AH}$.

\section{Results and Discussion}

\subsection{Bitterness AH Aample Analysis via Electronic Tongue}

The electronic tongue divides response range of the "minimum taste stimulation" and the "maximum taste stimulation" into 25 units according to Weber Fechner's law. Each unit represents that the concentration of the sample is changed by $20 \%$, but if the change lower than this unit, then normal people will not feel the difference between taste stimulation. The range of bitterness and astringency that humans can perceive is $0.00-25.00$ [21,22]. The linearity between the concentration of AH samples and sensor responses was observed. The results showed that the sensor responses increased with higher concentrations $(0.05-2 \mathrm{~g} / 100 \mathrm{~mL})$. Furthermore, the optimal concentration for determination was $0.1 \mathrm{~g}$ samples added to $100 \mathrm{~mL}$ of solution, which is detailed in Section 3.3.2. In this concentration, all of the values of the six taste factors were all within the range of 0.00-25.00. The results of repeatability and stability were as follows: precision - the relative standard deviations (RSD) of six taste factors' values were all less than 5\%; and, stability-less than $4 \%$. Thus, all of the results indicated that the electronic tongue measurements were reliable.

Four lipid membrane sensors of bitterness containing six taste factors were used in this study. Table 1 shows the bitterness values of these taste factors in 30 batches of AH samples. There are differences in the six taste factors among AH samples with different commercial classifications, the trends of the changes in these factors are not exactly the same. Thus, the radar curves of bitterness for 
all AH samples based on the six factors were constructed to comprehensively characterize the taste fingerprint information.

As shown in Figure 1, among the six taste factors, B-bitterness2 and Bitterness elicit the strongest responses. The corresponding values of B-bitterness 2 range from 0.93 to 8.53 , and Bitterness ranges from 1.38 to 3.86 (Table 1). Bitterness as the taste factor of the initial taste represents the taste of medicine in the mouth, whereas B-bitterness2 (i.e., aftertastes of mineral bitterness) represents the taste remaining in the mouth after swallowing. As shown in Table 1, the range and averages of the radar curve areas are as follows: AH leaf samples 7.790-13.449, average of 10.994; stem/leaf samples 2.857-11.067, average of 5.849; stem samples 1.421-2.932, average of 2.034. It can be seen that the bitterness levels are highest in the leaves, followed by stem/leaf combined, and lowest in the stems alone. Therefore, AH samples with different commercial classifications presented different taste fingerprint information, implying that the bitter values, especially the radar curves area, could be used as indexes for the determination of AH samples with commercial classification.

Table 1. Taste intensities of each taste factor in all AH samples (mean $\pm \mathrm{RSD}, n=3$ ) and the Radar curve area.

\begin{tabular}{|c|c|c|c|c|c|c|c|}
\hline Samples & B-Bitterness2 & Aftertaste-B & Aftertaste-A & H-Bitterness & Bitterness & Astringency & $\begin{array}{c}\text { Radar Curve } \\
\text { Area }\end{array}$ \\
\hline L1 & $6.83 \pm 0.01$ & $0.55 \pm 0.05$ & $0.19 \pm 0.03$ & $0.13 \pm 0.04$ & $3.86 \pm 0.03$ & $2.12 \pm 0.05$ & 11.713 \\
\hline L3 & $5.90 \pm 0.04$ & $0.47 \pm 0.05$ & $0.10 \pm 0.06$ & $0.04 \pm 0.07$ & $3.16 \pm 0.05$ & $1.66 \pm 0.03$ & 7.790 \\
\hline $\mathrm{L} 4$ & $6.57 \pm 0.02$ & $0.49 \pm 0.02$ & $0.15 \pm 0.07$ & $0.09 \pm 0.06$ & $3.39 \pm 0.06$ & $1.94 \pm 0.01$ & 9.931 \\
\hline L5 & $8.53 \pm 0.06$ & $0.41 \pm 0.05$ & $0.19 \pm 0.06$ & $0.24 \pm 0.04$ & $3.28 \pm 0.06$ & $1.99 \pm 0.03$ & 12.085 \\
\hline ST2 & $1.30 \pm 0.04$ & $0.30 \pm 0.05$ & $0.10 \pm 0.06$ & $0.08 \pm 0.08$ & $2.39 \pm 0.04$ & $1.62 \pm 0.05$ & 2.857 \\
\hline ST3 & $6.07 \pm 0.05$ & $0.34 \pm 0.03$ & $0.09 \pm 0.00$ & $0.21 \pm 0.07$ & $2.14 \pm 0.07$ & $1.21 \pm 0.09$ & 5.411 \\
\hline ST4 & $7.17 \pm 0.01$ & $0.42 \pm 0.01$ & $0.11 \pm 0.09$ & $0.25 \pm 0.06$ & $2.30 \pm 0.06$ & $1.23 \pm 0.09$ & 6.629 \\
\hline ST5 & $5.48 \pm 0.05$ & $0.47 \pm 0.05$ & $0.09 \pm 0.06$ & $0.17 \pm 0.06$ & $2.38 \pm 0.05$ & $1.15 \pm 0.04$ & 5.229 \\
\hline ST6 & $5.99 \pm 0.03$ & $0.30 \pm 0.04$ & $0.06 \pm 0.05$ & $0.18 \pm 0.06$ & $2.13 \pm 0.06$ & $1.14 \pm 0.05$ & 4.965 \\
\hline ST7 & $8.51 \pm 0.08$ & $0.46 \pm 0.07$ & $0.16 \pm 0.06$ & $0.24 \pm 0.09$ & $2.88 \pm 0.04$ & $1.51 \pm 0.05$ & 9.490 \\
\hline ST11 & $7.21 \pm 0.02$ & $0.30 \pm 0.05$ & $0.08 \pm 0.07$ & $0.16 \pm 0.06$ & $1.90 \pm 0.03$ & $1.07 \pm 0.04$ & 5.305 \\
\hline ST12 & $3.87 \pm 0.08$ & $0.41 \pm 0.05$ & $0.05 \pm 0.12$ & $0.19 \pm 0.03$ & $2.41 \pm 0.01$ & $1.23 \pm 0.05$ & 4.243 \\
\hline ST13 & $7.92 \pm 0.01$ & $0.43 \pm 0.04$ & $0.12 \pm 0.05$ & $0.24 \pm 0.07$ & $3.23 \pm 0.03$ & $1.91 \pm 0.01$ & 11.067 \\
\hline S1 & $0.93 \pm 0.05$ & $0.20 \pm 0.05$ & $0.05 \pm 0.10$ & $0.02 \pm 0.00$ & $2.07 \pm 0.05$ & $1.40 \pm 0.06$ & 1.922 \\
\hline $\mathrm{S} 2$ & $1.87 \pm 0.04$ & $0.18 \pm 0.06$ & $0.02 \pm 0.03$ & $0.11 \pm 0.05$ & $1.58 \pm 0.06$ & $1.03 \pm 0.08$ & 1.762 \\
\hline S3 & $2.08 \pm 0.07$ & $0.15 \pm 0.07$ & $0.01 \pm 0.11$ & $0.08 \pm 0.07$ & $1.38 \pm 0.03$ & $0.99 \pm 0.08$ & 1.667 \\
\hline $\mathrm{S} 4$ & $1.96 \pm 0.04$ & $0.24 \pm 0.08$ & $0.06 \pm 0.06$ & $0.09 \pm 0.06$ & $1.90 \pm 0.02$ & $1.14 \pm 0.07$ & 2.192 \\
\hline S5 & $3.03 \pm 0.03$ & $0.24 \pm 0.09$ & $0.04 \pm 0.13$ & $0.11 \pm 0.05$ & $1.42 \pm 0.08$ & $0.86 \pm 0.06$ & 2.046 \\
\hline S6 & $1.82 \pm 0.08$ & $0.23 \pm 0.07$ & $0.05 \pm 0.11$ & $0.07 \pm 0.09$ & $1.56 \pm 0.04$ & $0.81 \pm 0.05$ & 1.421 \\
\hline S7 & $4.09 \pm 0.01$ & $0.22 \pm 0.05$ & $0.03 \pm 0.10$ & $0.13 \pm 0.08$ & $1.38 \pm 0.03$ & $0.86 \pm 0.05$ & 2.509 \\
\hline S8 & $1.00 \pm 0.07$ & $0.41 \pm 0.05$ & $0.07 \pm 0.09$ & $0.02 \pm 0.00$ & $2.63 \pm 0.03$ & $1.37 \pm 0.03$ & 2.367 \\
\hline
\end{tabular}

L: represents leaf samples; ST: represents the mixed of stem and leaf samples; S: represents stem samples, $n=3$ means one prepared sample was repeatedly tested 3 times according to the procedure. 


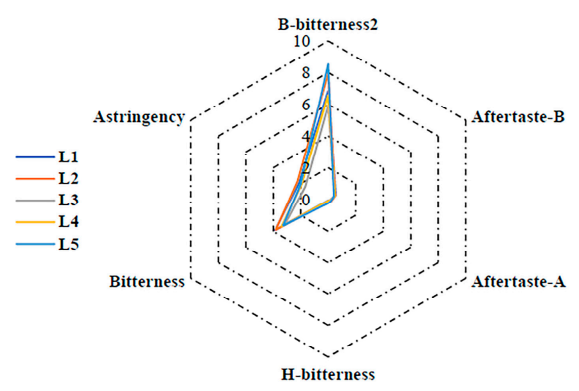

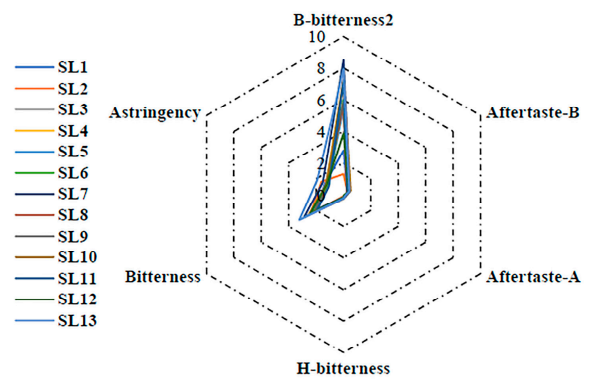

$\mathrm{B}$
A

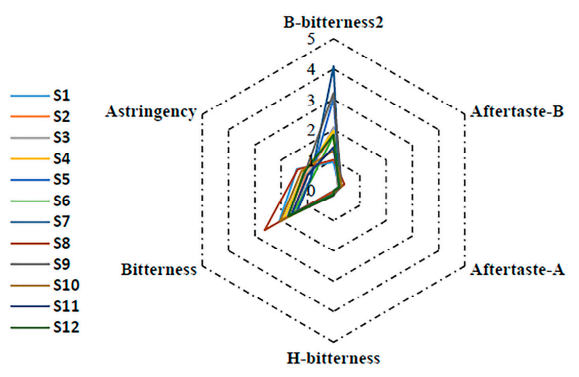

C

Figure 1. Radar curves of Andrographis Herba (AH) samples' bitterness for different commercial classifications. A: Leaf samples, B: Stem/Leaf samples, C: Stem samples.

\subsection{Principal Component Analysis of AH Samples' Bitterness}

In order to objectively and visually characterize the differences between $\mathrm{AH}$ samples with different commercial classifications, a Principal Component Analysis (PCA) was applied based on the values of the six taste factors. As an unsupervised pattern recognition method, PCA can visualize inherent clustering between different groups, which displays the internal structure of datasets in an unbiased way and decreases data dimensionality [23]. As shown in the PCA score three-dimensional (3D) plot (Figure 2), an overview of all data samples can be observed, which exhibited a clear grouping trend $\left(R^{2} X, 0.912 ; Q^{2}, 0.763\right)$ among the three classifications of samples. The $R^{2} X(0.912)$ and $Q^{2}$ (0.763) represented the PCA model, accounting for $91.2 \%$ data variance and a good predictive ability, respectively. This observation indicated that there were indeed differences in bitterness among $\mathrm{AH}$ samples with different commercial classifications.

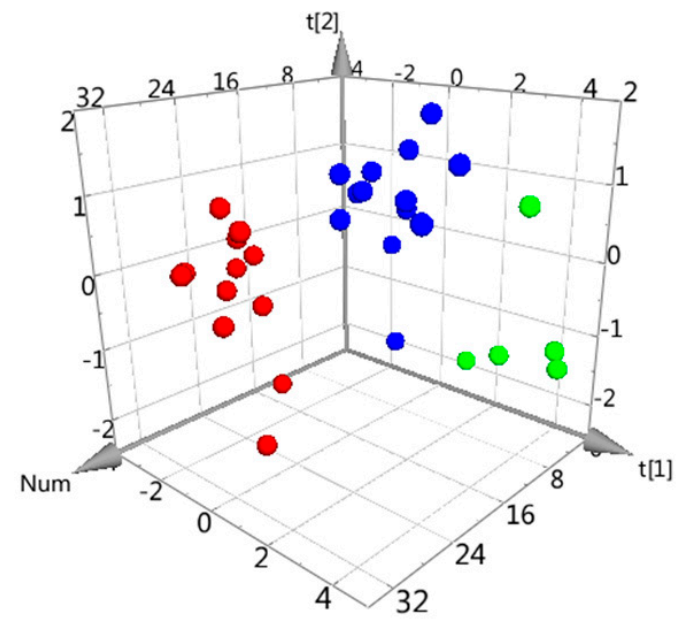

Figure 2. The Principal Component Analysis Three-Dimensional (PCA 3D) scores plot using AH bitterness data. ๑: Leaf, ๑: Stem/Leaf, ๑: Stem. 


\subsection{HPLC Fingerprint Analysis}

In order to obtain satisfactory efficiency, three extraction methods (refluxing, ultrasonic, and cold-macerating extraction), a range of extraction solvent concentrations ( $20 \%$ methanol, $40 \%$ methanol, $60 \%$ methanol, $80 \%$ methanol, and 100\% methanol) and extraction times $(0.2 \mathrm{~h}, 0.3 \mathrm{~h}, 0.5 \mathrm{~h}, 1 \mathrm{~h})$ were compared and optimized using univariate tests. The results indicated that there are no obvious differences in the three aforementioned extraction methods. Thus, the most convenient method of ultrasonic extraction was selected. It was found that $40 \%$ methanol was the most efficient extraction solvent among the different concentrations based on the main peak areas in the chromatogram. In addition, it was demonstrated that most components could be extracted completely within $0.5 \mathrm{~h}$. In summary, samples were prepared by ultrasonic extraction with $50 \mathrm{~mL}$ of $40 \%$ methanol for $0.5 \mathrm{~h}$.

As shown in Figure 3, nine common peaks in the chromatogram were selected as the markers for the fingerprints method validation. The relative retention time (RRT) and relative peak area (RPA) of these peaks were calculated for estimation of precision, repeatability, and stability, and the results were as follows: precision - the relative standard deviations (RSD) of RRT and RPA were found not to exceed $0.03 \%$ and $2.68 \%$, respectively; repeatability-below $0.07 \%$ and $3.14 \%$, respectively; and, stability-less than $0.07 \%$ and $3.55 \%$, respectively. Thus, all results indicated that the HPLC measurements were stable and under control.

30 batches of $\mathrm{AH}$ samples with different commercial classifications were analyzed, and their corresponding chromatographic fingerprints were aligned and matched using the Similarity Evaluation System for chromatographic fingerprint of TCM (Figure 3). Furthermore, the common peaks of 2,6, 7, and 8 were identified as andrographolide, neoandrographolide, 14-deoxyandrographolide, and dehydroandrographolide, respectively, by comparison with reference compounds that were based on the ultraviolet spectrum and retention time. With an overview of all samples in the chromatographic fingerprints, the fingerprints' characteristics vary depending on the commercial classification of the samples. For example, peak 2 in the chromatogram was obviously the highest within the leaf samples, followed by stem and leaf mixed samples, and was lowest in the stem samples. Therefore, in order to discover the key bitter substances from the fingerprint, we further took a correlation analysis between the radar curve areas of the bitter substances and the nine common peak areas of the chromatographic fingerprints.

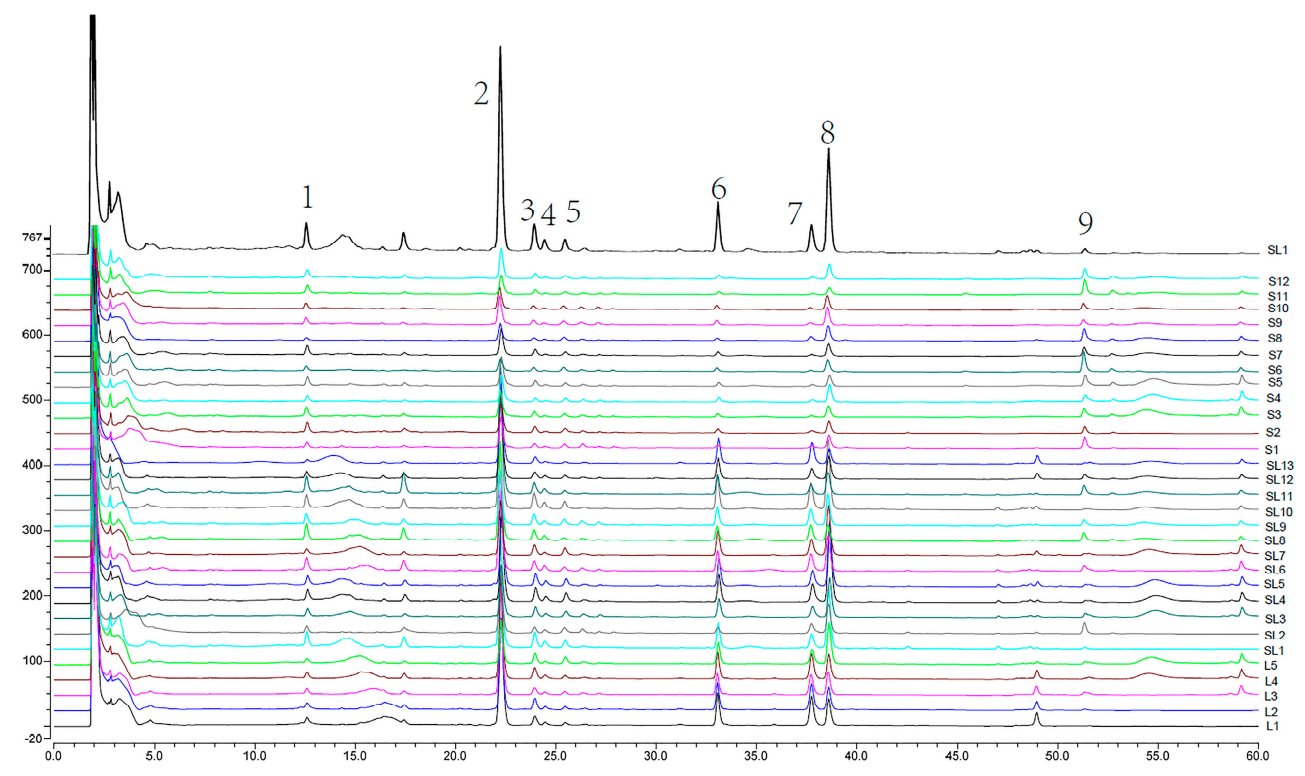

Figure 3. Chromatographic fingerprints of all AH samples. (L1-L5 are leaf samples; SL1-SL13 are stem and leaf mixed samples; S1-S12 are stem samples. Peaks 2,6,7,8 are andrographolide, neoandrographolide, 14-deoxyandrographolide, and dehydroandrographolide, respectively). 


\subsection{Spearman Correlation Analysis}

In this study, the Spearman correlation analysis was performed to find the key bitter compounds using the Software SPSS21.0 (SPSS Inc., Chicago, IL, USA). The correlation coefficients between bitter (the radar curve area) and common peaks are summarized in Table 2. These correlations are depicted visually in Figure 4. The closer the absolute value of the correlation coefficient is to 1 , the more significant the correlation. Generally, if the absolute value of the correlation coefficient was more than 0.5 , it indicated a reliable positive or negative correlation $(p<0.01)$.

Table 2. Correlation Coefficients between bitter (radar curve area) and the common peaks in HPLC fingerprint.

\begin{tabular}{cccc}
\hline Common Peaks & Radar Curve Area & Common Peaks & Radar Curve Area \\
\hline 1 & $\rho=-0.125(p=0.510)$ & 6 & $\rho=0.854(p<0.01)$ \\
2 & $\rho=0.725(p<0.01)$ & 7 & $\rho=0.890(p<0.01)$ \\
3 & $\rho=0.729(p<0.01)$ & 8 & $\rho=0.691(p<0.01)$ \\
4 & $\rho=0.629(p<0.01)$ & 9 & $\rho=-0.826(p<0.01)$ \\
5 & $\rho=-0.014(p=0.942)$ & & \\
\hline
\end{tabular}

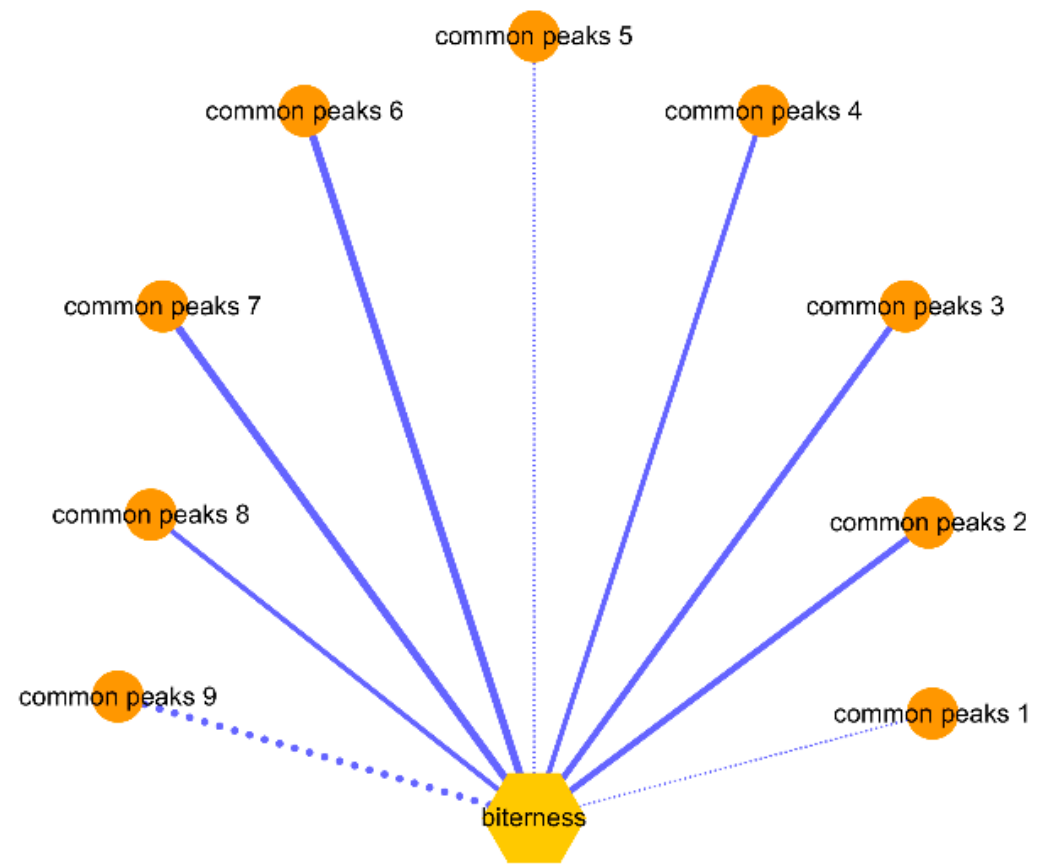

Figure 4. Correlation network between bitterness (radar curve area) and the common peaks in HPLC fingerprint. Visualization of data concentrated on the correlations between chemical constituents in relation to bitterness (radar curve area). The negative correlations are indicated with dots lines, and positive correlations are indicated with solid lines; thicker lines indicate a stronger correlation. The length of each line has no meaning.

Of the nine common peaks, the areas of peaks $2,3,4,6,7$, and 8 showed a highly positive correlation with the bitter compounds (the radar curve area), and the corresponding correlation coefficients were $0.725,0.7290 .629,0.854,0.890$, and 0.691 , respectively. The area of peak 9 showed a highly negative correlation with bitterness, and the correlation coefficient is -0.826 . In addition, there were no significant correlations observed between the areas of peaks 1,5, and the radar curve area of bitter substances. In summary, the more bitter the AH, the higher relative contents of andrographolide (peak 2), neoandrographolide (peak 6), 14-deoxyandrographolide (peak 7), dehydroandrographolide (peak 8), and peaks 3 and 4 . In contrast, the more bitter the $\mathrm{AH}$, the lower the relative contents of peak 9. 
It was known that $\mathrm{AH}$ as a representative TCM with bitter properties is often used for antibacterial and anti-inflammatory remedies in the clinic [24]. The bitter-related substances of andrographolide (peak 2), neoandrographolide (peak 6), 14-deoxyandrographolide (peak 7), and dehydroandrographolide (peak 8) have also been reported with obvious antibacterial and anti-inflammatory effects [3]. Therefore, the bitterness that was detected by the electronic tongue can be used not only to distinguish AH samples with different commercial classifications, but also to reflect the levels of AH effective ingredients. In addition, the results suggested that the other unknown bitter-related substances (Peak 3,4) may also have bitter activities, such as antibacterial and anti-inflammatory.

\section{Materials and Methods}

\subsection{Instruments and Chemicals}

Bitterness measurements were performed using electronic tongues TS-5000Z (Insent Inc., Atsugi, Japan). HPLC analyses for chromatographic fingerprints were performed with a Dionex U-3000 series (Shanghai, China). HPLC-grade methanol and acetonitrile were supplied by Thermo Fisher Scientific Inc. (Shanghai, China). Deionized water was purchased (Wahaha, China). 20\% methanol, $40 \%$ methanol, $60 \%$ methanol, and $80 \%$ methanol were prepared by dilution of absolute methanol with deionized water. The reference compounds andrographolide, dehydroandrographolide, 14-deoxyandrographolide, and neoandrographolide (purity $>95 \%$ for all) were purchased from Chengdu Chroma-Biotechnology Co., Ltd. (Sichuan, China). Potassium chloride (analytical grade), tartaric acid (ISO), ethanol (99.8\%), hydrochloric acid (36-38\%), silver chloride (analytical grade), and potassium hydroxide (ISO) were purchased from Fajiede Chemical Reagent Inc. (Beijing, China).

\subsection{AH Sample Collection}

30 batches of $\mathrm{AH}$ with different commercial classifications were collected from various provinces in China (Table 3), including their production areas (such as Guangdong, Guangxi, etc.). Samples were denoted, as follows: leaves, L1 to L5; mixed stems and leaves, SL1 to SL13; and, stems, S1 to S12. Three typical AH samples with different specification are shown as Figure 5. In the mixed samples, the ratio of stems to leaves is about $20-50 \%$. The quantity and proportion of three kinds of samples collected represent the actual situation of AH on the market. The samples were identified by Professor Zhuju Wang at the Institute of Chinese Materia Medica, China Academy of Chinese Medical Sciences. All samples were stored in a dry, constant environment to minimize any changes through degradation, and the voucher specimens were deposited in our laboratory.

Table 3. AH collection information.

\begin{tabular}{cccccc}
\hline Samples & Origin & Collection Parts & Samples & Origin & Collection Parts \\
\hline L1 & Anhui & Leaf & SL11 & Guangdong & Stem/Leaf \\
L2 & Anhui & Leaf & SL12 & Anhui & Stem/Leaf \\
L3 & Anhui & Leaf & SL13 & Anhui & Stem/Leaf \\
L4 & Anhui & Leaf & S1 & Jiangxi & Stem \\
L5 & Anhui & Leaf & S2 & Anhui & Stem \\
SL1 & Jiangxi & Stem/Leaf & S3 & Guangxi & Stem \\
SL2 & Jiangxi & Stem/Leaf & S4 & Guangxi & Stem \\
SL3 & Guangxi & Stem/Leaf & S5 & Guangxi & Stem \\
SL4 & Fujian & Stem/Leaf & S6 & Guangxi & Stem \\
SL5 & Guangxi & Stem/Leaf & S7 & Guangdong & Stem \\
SL6 & Guangxi & Stem/Leaf & S8 & Guangdong & Stem \\
SL7 & Guangxi & Stem/Leaf & S9 & Sichuan & Stem \\
SL8 & Guangdong & Stem/Leaf & S10 & Sichuan & Stem \\
SL9 & Guangdong & Stem/Leaf & S11 & Jiangsu & Stem \\
SL10 & Guangdong & Stem/Leaf & S12 & Jiangsu & Stem \\
\hline
\end{tabular}




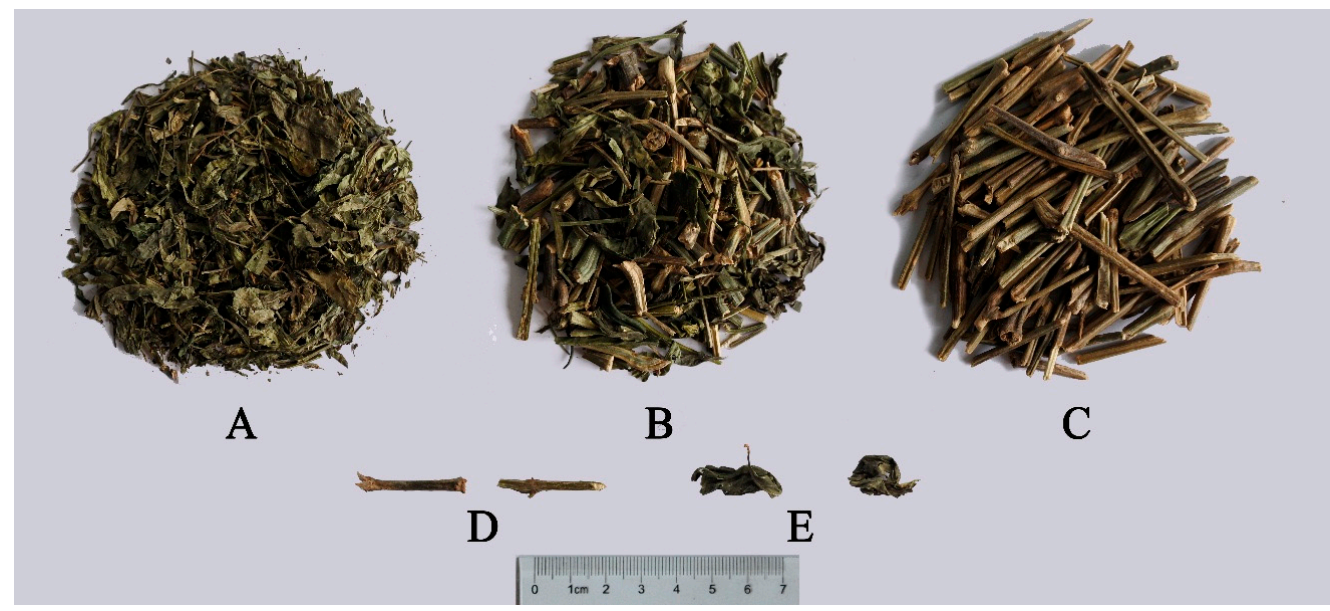

Figure 5. Three typical AH samples with different specification. A: leaf sample; B: a mixture of leaf and stem sample; C: stem sample; D: stem; E: leaf.

\subsection{Electronic Tongue Methods}

\subsubsection{Electronic Tongue Measurement Principle, Steps, and Conditions}

The measurement principle of electronic tongue TS-5000Z is potentiometric, during measurement, $\mathrm{mV}$ values are recorded and no absolute taste values are obtained. The artificial lipid membrane sensor probe is composed of silver-wire electrode, the surface of which is coated with $\mathrm{Ag} / \mathrm{AgCl}$, with a sensor body made of polypropylene, and lipid membranes made by mixing lipids (which play an important role in taste sensing) with a polymer [20]. Before each measurement, a sensor check was performed to ensure that the sensors were working in the correct voltage range. During one measurement, every sample was measured four times. For data interpretation, the last three runs were used to enable conditioning of the lipid membranes to the sample solutions and to ensure data stability.

Every sample measurement starts with a cleaning procedure. After cleaning, the stability of the lipid membrane potential was controlled by measuring the potential of the reference solution $(\mathrm{Vr})$. When the sensor response is stable during $30 \mathrm{~s}$ of measurement (deviation smaller than $0.5 \mathrm{mV}$ ), the sample solution was measured for $30 \mathrm{~s}$ (Vs). Electrical potential changes (R) between Vr and Vs was called the relative potential and used to calculate the initial tastes. After a short cleaning procedure $(3 \mathrm{~s}$, two times), the membrane potential is measured again in reference solution for $30 \mathrm{~s}\left(\mathrm{Vr}^{\prime}\right)$, the change in electric potentials between $\mathrm{Vr}$ and $\mathrm{Vr}^{\prime}$ is called the change of membrane potential that is caused by adsorption (CPA) value and is used to calculate the aftertaste. The CPA value results from the measurement of the adsorption of substances, which are not removed by the short cleaning procedure from the lipid membrane. The electronic tongue detects the membrane potential, and then converts the potential value into a taste value according to Weber Fechner's law that the intensity of the perception is proportional to the logarithm of stimulus intensity [20].

The electronic tongue TS-5000Z is equipped with up to eight lipid membrane sensors and a reference electrode. The bitter taste is usually represented by three different sensors, labeled C00, BT0, and AN0. In this experiment, except for the three bitterness sensors, an astringency sensor AE1 (auxiliary measurement) was also used to measure bitterness of AH. Especially, the sensors of C00 and AE1 can measure two taste factors, one of which is the initial taste representing the initial taste of medicine in the mouth, whereas the other is the aftertaste representing the taste remaining in the mouth after swallowing (Table 4). Therefore, a total of six values were obtained to characterize the bitterness of the AH samples. The $\mathrm{pH}$ range of the sample to be tested should be controlled within 2-8, and all measurement procedures were carried out at the room temperature $\left(23-26^{\circ} \mathrm{C}\right)$. 
Table 4. Taste information represented by four taste sensors.

\begin{tabular}{ccc}
\hline \multirow{2}{*}{ Sensor Probes } & \multicolumn{2}{c}{ Taste Information } \\
\cline { 2 - 3 } & Initial Value & Aftertaste Value \\
\hline C00 & Bitterness & Aftertaste of anionic bitterness (aftertaste-B) \\
BT0 & - & Aftertaste of cationic bitterness (H-bitterness) \\
AN0 & - & Aftertaste of mineral bitterness (B-bitterness2) \\
AE1 & Astringency & Aftertaste of astringency (aftertaste-A) \\
\hline \multicolumn{2}{c}{ “-“ indicates no relative value. }
\end{tabular}

Potassium chloride and tartaric acid were dissolved in distilled water as reference solution at concentrations of $30 \mathrm{mmol} / \mathrm{L}$ and $0.3 \mathrm{mmol} / \mathrm{L}$, respectively, for sensor conditioning and cleaning. For washing the different charged lipid membranes of the sensors, two solutions were prepared: $100 \mathrm{mmol} / \mathrm{L}$ hydrochloric acid dissolved in 30\% ethanol (made by dilution of absolute ethanol with deionized water) for negatively charged membranes (BT0, AN0); $100 \mathrm{mmol} / \mathrm{L}$ potassium chloride and $10 \mathrm{mmol} / \mathrm{L}$ potassium hydroxide both dissolved in $30 \%$ ethanol for positively charged membranes (AE1, C00). A solution of $3.33 \mathrm{mmol} / \mathrm{L}$ potassium chloride in saturated silver chloride was used for sensors and reference electrodes as an inner solution. The sensors were embedded in reference solution for one day prior to being used for measurements.

\subsubsection{Sample Preparation for the Electronic Tongue}

Each of the dried samples was crushed into a powder with a pulverizer for $2 \mathrm{~min}$ and was passed through a $65 \mu \mathrm{m}$-mesh sieve. Each sample powder was weighed accurately at $0.1 \mathrm{~g}$, and $100 \mathrm{~mL}$ of $10 \mathrm{mmol} / \mathrm{L}$ potassium chloride solution was added to increase the conductivity of the solution. After ultrasonication for $30 \mathrm{~min}$, the sample was filtered through gauze and the filtrate was placed in an electronic beaker for testing.

\subsubsection{Electronic Tongue Methodology Validation}

Methodology validation of electronic tongue was performed for verifying the use of the electronic tongue in AH samples. The linearity was investigated to evaluate the relationship between the sensor responses and the concentrations by measuring the same sample with five different concentrations ( $0.05 \mathrm{~g}, 0.1 \mathrm{~g}, 0.5 \mathrm{~g}, 1 \mathrm{~g}$ and $2 \mathrm{~g}$ of AH powders in $100 \mathrm{~mL}$ of $10 \mathrm{mmol} / \mathrm{L}$ potassium chloride solution). Five parallelly prepared samples from Sample L1 (Table 1) were determined according to the procedure to test repeatability. The sample stability was determined by analyzing a single prepared sample that was stored at room temperature for $0,1,2,4$, and $8 \mathrm{~h}$. The RSDs $\%$ of the sensor responses were all calculated to estimate repeatability and stability.

\subsection{HPLC Fingerprint Methods}

\subsubsection{Chromatographic Conditions}

All of the HPLC analyses were performed with a Dionex U-3000 series equipped with a SR-3000 Solvent Rack, a LPG-3400SDN Quaternary Pump, a WPS-3000SL Auto sampler, a TCC-3000RS Column compartment, a DAD-3000RS detector, and a Chromeleon 7 chromatography workstation (Thermo Fisher Scientific, Waltham, MA, USA). An Agilent ZORBAX Extend-C18 column $(4.6 \mathrm{~mm} \times 250 \mathrm{~mm}, 5 \mu \mathrm{m})$ was used. The mobile phase consisted of acetonitrile (A) and water (B). The gradient program was developed as follows: $15-28 \%$ A for $0-22 \mathrm{~min}, 28-37 \%$ A for $22-35 \mathrm{~min}$, $37-50 \%$ A for $35-45 \mathrm{~min}$, and $50-75 \%$ A for $45-60 \mathrm{~min}$. The flow rate was maintained at $1.0 \mathrm{~mL} / \mathrm{min}$ and the column temperature at $30^{\circ} \mathrm{C}$. The injection volume was $10 \mu \mathrm{L}$ and the detective wavelength was selected at $205 \mathrm{~nm}$. 


\subsubsection{HPLC Sample Preparation}

Each sample powder $(0.5 \mathrm{~g})$ was added to a $100 \mathrm{~mL}$ Erlenmeyer flask with $50 \mathrm{~mL} 40 \%$ methanol, and the flask was accurately weighed. Following soaking for $1 \mathrm{~h}$ and ultrasonic extraction for $30 \mathrm{~min}$, the sample mixture was weighed again and any solvent that was lost in the process was added after being cooled to room temperature. Subsequently, the mixture was filtered through a $0.22 \mu \mathrm{m}$ membrane filter. Finally, $10 \mu \mathrm{L}$ aliquots from the filtrate were subjected to HPLC analysis. Stock solutions of the four reference compounds of andrographolide, dehydroandrographolide, 14-deoxyandrographolide and neoandrographolide—of about $0.2 \mathrm{mg} / \mathrm{mL}$-were prepared in methanol and stored at $4{ }^{\circ} \mathrm{C}$ for later analysis.

\subsubsection{HPLC Methodology Validation}

All AH samples were prepared, as described in Section 3.4.2. The precision of the chromatographic method was established by analyzing the same sample solution five times within one day. Precision was expressed as the RSD\% of repeated measurements. The sample stability was determined by analyzing a single sample solution that was stored at room temperature for $0,2,4,8,12$, and $24 \mathrm{~h}$. Repeatability was determined by analyzing five separate samples from the same source. The RRT and RPA of each of the common peaks were calculated to estimate precision, stability, and repeatability.

\subsection{Data Analysis}

\subsubsection{PCA Analysis for Electronic Tongue Data}

The raw data was saved as Common Executable Format (CEF)-files (the rows represent observed samples, the columns represent the variables of bitterness values) and imported into the software of SIMCA-P (Umetrics AB, Umea, Sweden) for PCA employing the Nonlinear Iterative Partial Least Square (NIPALS) algorithm. PCA was performed on the raw data and the pretreatment of the data was performed by UV scaling. PCA, as an unsupervised pattern recognition pattern, generate new original variables, but shows linear combinations of them and simultaneously capture most features of the original data. Thus, PCA decreases the dimensionality of data and could be used to visualize inherent clustering between the AH samples. The score values plots for the first two or three PCs (PC1, PC2, and PC3) are often used to visually represent the characteristics of the samples. The parameters of the modeling, $R^{2}$ and $Q^{2}$ values in PCA, can explain the quality of the fitting model. $R^{2}$ is the percent of variation of the training set- $X$ with PCA- explained by the model. $R^{2}$ is a measure of fit, i.e., how well the model fits the data. Later, $R^{2} X$ is the fraction of the variation of the $X$ variables explained by the model. A large $R^{2}$ (close to 1 ) is a necessary condition for a good model, but it is not sufficient. $Q^{2}$ is the percent of variation of the training set- $X$ with PCA-predicted by the model according to cross validation. $Q^{2}$ indicates how well the model predicts new data. A large $Q^{2}\left(Q^{2}>0.5\right)$ indicates good predictivity $[25,26]$.

\subsubsection{Fingerprint Data Processing}

The raw HPLC chromatographic data of the 30 tested samples were integrated automatically and exported as *. AIA format files for further processing. Then, all of these files were imported into the Similarity Evaluation System for TCM chromatographic fingerprinting (Version 2004 A; Committee for the Pharmacopoeia of PR China.). One sample was randomly selected as a reference to generate the template. Subsequently, all of the samples were automatically aligned on the basis of this template and the reference peaks. For the chromatograms, which were to be arranged in a line, reference peaks were first aligned to those in the template, and the other peaks were subsequently lined up on the basis of the nearest reference peak in the chromatogram. For further analysis, the retention time and peak area of all aligned peaks were calculated simultaneously and can be exported as an excel file for further statistical analysis. 


\subsubsection{Correlation Analysis}

Input the six taste factors' value into excel to generate a radar curve, and the area of the radar curve is used to comprehensively represent AH bitterness. The Spearman correlation coefficient is the most commonly used measure of monotone association and it is usually suggested for non-normally distributed data [27]. The data distribution was not normal by the Shapiro-Wilk test and then the Spearman's rank correlation $(\rho)$ was used to quantify the correlation between the radar curve areas and the common peaks in fingerprints (SPSS version 21.0). Significant correlations were defined as Spearman's $|\rho|>0.5$ and $p<0.01$, respectively. Thereafter, Cytoscape version 3.7.0 (www.cytoscape. org) was used to draw a network view to visualize these correlations [28,29].

The Radar curve area was calculated, as follows:

$$
\text { Radar curve area }=\sqrt{3} / 4 \times(a b+b c+c d+d e+e f+f a)
$$

where $a$ is B-bitterness2, $b$ is Aftertaste-B, $c$ is Aftertaste-A, $d$ is H-bitterness, and $e$ and $f$ are Bitterness and Astringency, respectively (Table 2).

\section{Conclusions}

In this study, electronic tongue technology was firstly applied to assess the bitterness of $\mathrm{AH}$. Based on the six taste factors, the PCA score 3D plot (Figure 2) exhibited a clear grouping trend $\left(R^{2} X\right.$, 0.912; $\mathrm{Q}^{2}, 0.763$ ) among the three different commercial classifications of samples: leaves, stems, and mixtures of both. The results implied that electronic tongue had the ability to distinguish the bitterness among different commercial classifications. Six compounds (peaks 2, 3, 4, 6, 7, and 8) with positive correlations to bitterness were discovered by Spearman correlation analysis. Furthermore, the peaks 2, 6, 7, and 8 were identified as andrographolide, neoandrographolide, 14-deoxyandrographolide, and dehydroandrographolide, respectively. In summary, detecting bitterness via electronic tongue technology could evaluate the quality of $\mathrm{AH}$ samples rapidly and efficiently.

Author Contributions: L.T. and Z.W. conceived and designed the experiments; X.Z. and L.T. performed the experiments; X.Z. and X.Y. analyzed the data; X.Z. and H.W. wrote the paper; Y.L., H.L., H.Y., X.L. and Z.L. reviewed the paper. All authors read and approved the final manuscript.

Funding: This research was funded by the National standardization project of TCM (No. ZYBZH-C-JX-41) and the Key Research and Development Plan of Shandong Province in 2018 (No. 2018CXGC1305).

Acknowledgments: The authors are grateful to Ensoul Technology Ltd., Beijing for providing the electronic tongue measurements as well as technical support. And the authors are grateful to Enago for providing language assistance and grammar check.

Conflicts of Interest: The authors have no conflicts of interests to declare.

\section{References}

1. Sareer, O.; Ahad, A.; Umar, S. Prophylactic and lenitive effects of Andrographis Paniculata against common human ailments: An exhaustive and comprehensive reappraisal. J. Pharm. Res. Opin. 2012, 10, 138-162.

2. Sareer, O.; Ahmad, S.; Umar, S. Andrographis paniculata: A critical appraisal of extraction, isolation and quantification of andrographolide and other active constituents. Nat. Prod. Res. 2014, 28, 2081-2101. [CrossRef] [PubMed]

3. Zhang, X.; Tang, L.Y.; Wu, H.W.; Xu, M.Y.; Guo, R.X.; Yu, X.K.; Lu, Y.Q.; Luo, H.Y.; Liu, D.F.; Wang, Z.J.; et al. The newest research progresses of Andrographis Paniculate. Chin. J. Exp. Tradit. Med. Form. 2018, 24, 96-108.

4. Committee for the Pharmacopoeia of China. Pharmacopoeia of China; Part I; China Medical Science and Technology Press: Beijing, China, 2015; pp. 268-269.

5. Liu, C.X.; Zhang, T.J.; He, X.; Chen, C.Q.; Xu, J.; Tian, C.W. Study on chemistry and biology based on five-tastes and function-efficacy of Chinese materia medica with invigorating blood circulation and eliminating blood stasis. Chin. Tradit. Herbal Drugs 2015, 46, 615-624. 
6. Tang, D.C. Science of Chinese Materia Medica, 1st ed.; Shanghai University of Traditional Chinese Medicine Press: Shanghai, China, 2003; p. 5. ISBN 7-810810-658659.

7. Bartoshuk, L.M. Comparing sensory experiences across individuals: Recent psychophysical advances illuminate genetic variation in taste perception. Chem. Senses 2000, 25, 447-460. [CrossRef] [PubMed]

8. Huang, J.Y.; Liu, X.L.; Zhou, S.P.; Tong, L.; Ding, L. Chemical fingerprinting of Andrographis Paniculata and quantitative analysis of four diterpenoids by UPLC-PDA. Chin. J. Chin. Mater. Med. 2014, 21, 4240-4245.

9. Zhang, X.; Wu, H.W.; Yu, X.K.; Lu, Y.Q.; Luo, H.Y.; Yang, H.J.; Xu, M.Y.; Guo, R.X.; Li, Z.Y.; Tang, L.Y.; et al. Quality evaluation of Andrographis Paniculate based on electronic-eye technique. Chin. J. Exp. Tradit. Med. Form. 2018, 17, 1-7.

10. Woertz, K.; Tissen, C.; Kleinebudde, P.; Breitkreutz, J. Taste sensing systems (electronic tongues) for pharmaceutical applications. Int. J. Pharm. 2011, 417, 256-271. [CrossRef]

11. Tahara, Y.; Toko, K. Electronic Tongues-A Review. IEEE Sens. J. 2013, 13, 3001-3011. [CrossRef]

12. Imamura, T.; Toko, K.; Yanagisawa, S.; Kume, T. Monitoring of fermentation process of miso (soybean paste) using multichannel taste sensor. Sensor. Actuat. B Chem. 1996, 37, 179-185. [CrossRef]

13. Eckert, C.; Lutz, C.; Breitkreutz, J.; Woertz, K. Quality control of oral herbal products by an electronic tongue-Case study on sage lozenges. Sensor. Actuat. B Chem. 2011, 156, 204-212. [CrossRef]

14. Kataoka, M.; Tokuyama, E.; Miyanaga, Y.; Uchida, T. The taste sensory evaluation of medicinal plants and Chinese medicines. Int. J. Pharm. 2008, 351, 36-44. [CrossRef]

15. Woertz, K.; Tissen, C.; Kleinebudde, P.; Breitkreutz, J. Rational development of taste masked oral liquids guided by an electronic tongue. Int. J. Pharm. 2010, 400, 114-123. [CrossRef] [PubMed]

16. Mohamed-Ahmed, A.H.A.; Soto, J.; Ernest, T.; Tuleu, C. Non-human tools for the evaluation of bitter taste in the design and development of medicines: A systematic review. Drug. Discov. Today 2016, 21, 1170-1180. [CrossRef] [PubMed]

17. Woertz, K.; Tissen, C.; Kleinebudde, P.; Breitkreutz, J. A comparative study on two electronic tongues for pharmaceutical formulation development. J. Pharm. Biomed. Anal. 2011, 55, 272-281. [CrossRef] [PubMed]

18. Ono, N.; Miyamoto, Y.; Ishiguro, T.; Motoyama, K.; Hirayama, F.; Iohara, D.; Seo, H.; Tsuruta, S.; Arima, H.; Uekama, K. Reduction of bitterness of antihistaminic drugs by complexation with $\beta$-cyclodextrins. J. Pharm. SCI-US 2011, 100, 1935-1943. [CrossRef] [PubMed]

19. Liu, F.; Xie, D.S.; Liu, H.M.; Fang, C.X.; Wei, C.; Guo, L.; Fu, C.M. Identification of Fritillaria cirrhosa powder and its adulterated products by electronic tongue technology. Chin. Tradit. Pat. Med. 2017, 39, 1977-1979.

20. Anjiki, N.; Hosoe, J.; Fuchino, H.; Kiuchi, F.; Sekita, S.; Ikezaki, H.; Mikage, M.; Kawahara, N.; Goda, Y. Evaluation of the taste of crude drug and Kampo formula by a taste-sensing system (4): Taste of Processed Aconite Root. J. Nat. Med. 2011, 65, 293-300. [CrossRef]

21. Fu, Z.H.; Li, S.J.; Hu, H.H.; Liu, P.; Sun, M.L. Taste comparison of unprocessed and processed Siegesbeckiae puescens based on electronic tongue. Chin. Tradit. Herbal Drugs 2017, 48, 673-680.

22. Kobayashi, Y.; Habara, M.; Ikezazki, H.; Chen, R.; Naito, Y.; Toko, K. Advanced taste sensors based on artificial lipids with global selectivity to basic taste qualities and high correlation to sensory scores. Sensors 2010, 10, 3411-3443. [CrossRef]

23. Letellier, T.; Durrieu, G.; Malgat, M.; Rossignol, R.; Antoch, J.; Deshouillers, J.M.; Coquet, M.; Lacombe, D.; Netter, J.C.; Pedespan, J.M.; et al. Statistical analysis of mitochondrial pathologies in childhood: Identification of deficiencies using principal component analysis. Lab. Investig. 2000, 80, 1019-1030. [CrossRef] [PubMed]

24. Liang, X.G.; Wu, F.; Wang, Y.J.; Fu, Z.; Wang, Y.; Feng, Y.; Liang, S. Research on bitter substances of traditional Chinese medicine Coptis chinensis based on electronic tongue technology. Chin. J. Chin. Mater. Med. 2014, 39, 3326-3329.

25. Wu, H.W.; Wang, P.; Liu, M.T.; Tang, L.Y.; Fang, J.; Zhao, Y.; Zhang, Y.; Li, D.F.; Xu, H.Y.; Yang, H.J. A ${ }^{1} \mathrm{H}-\mathrm{NMR}$-Based Metabonomic Study on the Anti-Depressive Effect of the Total Alkaloid of Corydalis Rhizoma. Molecules 2015, 20, 10047-10064. [CrossRef] [PubMed]

26. Liu, M.T.; Liu, X.; Wang, H.P.; Xiao, H.B.; Fang, J.; Tang, L.Y.; Li, D.F.; Zhang, Y.; Wu, H.W.; Yang, H.J. Metabolomics study on the effects of Buchang Naoxintong capsules for treating cerebral ischemia in rats using UPLC-Q/TOF-MS. J. Ethnopharmacol. 2016, 180, 1-11. [CrossRef] [PubMed]

27. Nian Shong Chok, M.S. Pearson's Versus Spearman's and Kendall's Correlation Coefficients for Continuous Data. Master's Thesis, University of Pittsburgh, Auckland, PA, USA, 2010. 
28. Sun, M.M.; Li, L.; Wang, M.; van Wijk, E.; He, M.; van Wijk, R.; Koval, S.; Hankemeier, T.; van der Greef, J.; Wei, S.L. Effects of growth altitude on chemical constituents and delayed luminescence properties in medicinal rhubarb. J. Photochem. Photobiol. B 2016, 162, 24-33. [CrossRef] [PubMed]

29. Franco, G.; De Nunzio, C.; Leonardo, C.; Tubaro, A.; Ciccariello, M.; De Dominicis, C.; Miano, L.; Laurenti, C. Ultrasound assessment of intravesical prostatic protrusion and detrusor wall thickness-new standards for noninvasive bladder outlet obstruction diagnosis? J. Urol. 2010, 183, 2270-2274. [CrossRef]

Sample Availability: Smples of the compounds are available from the authors.

(C) 2018 by the authors. Licensee MDPI, Basel, Switzerland. This article is an open access article distributed under the terms and conditions of the Creative Commons Attribution (CC BY) license (http://creativecommons.org/licenses/by/4.0/). 